\title{
THE DOMAIN OF UNIVALENCE OF CERTAIN FAMILIES OF RATIONAL FUNCTIONS
}

\author{
A. W. GOODMAN
}

\begin{abstract}
The Cakalov-Distler Theorem gives the domain of univalence for the family of all rational functions for which all the poles are simple, all the poles lie in a prescribed convex set, and all have positive residue. Here we extend this theorem to allow simple poles with negative residues. We include several open problems.
\end{abstract}

1. Introduction. Let $K$ be a closed disk in the plane and let $\mathscr{F}_{1}$ be the set of all functions of the form

$$
f(z)=\sum_{k=1}^{n} \frac{A_{k}}{z-a_{k}},
$$

where each $a_{k}$ is in $K$ and $A_{k} \geqslant 0$ for all $k$ with at least one $A_{k} \neq 0$.

Cakalov [1], [2] found the domain of univalence of the family $\mathscr{F}_{1}$. Here the domain of univalence of a family $\mathscr{F}$ means the maximal domain $D$ such that every $f(z)$ in $\mathscr{F}$ is univalent in $D$. Let $C$ be the Cakalov disk of $K$, that is, $C$ and $K$ have the same center, and the radius of $C$ is $\sqrt{2}$ times the radius of $K$. Then we have

TheOREM A (ČAKALOv). The domain of univalence of the family $\mathscr{F}_{1}$ (described above) is the complement of the Ćakalov disk of $K$.

We replace the disk $K$ by a line segment $I$ and let $\mathscr{F}_{2}$ be the family of functions of the form (1) where each $a_{k}$ is in $I$ and the $A_{k}$ satisfy the conditions stated earlier. Then according to Cakalov [1], [2] we have

THEOREM B. The domain of univalence of $\mathscr{F}_{2}$ is the complement of the closed disk $C$ that has I as its diameter.

Distler [3] replaced the disk and the interval by an arbitrary closed and convex set $K$ and considered $\mathscr{F}_{3}$, the family of functions of the form (1) where each $a_{k}$ is in $K$ and the $A_{k}$ satisfy the conditions stated earlier. The director set of $K$ consists of all points of $K$ together with those points $z$ at which $K$ subtends an angle $\eta \geqslant \pi / 2$. Then we have

TheOREM C (Distler). If $C$ is the director set of $K$, a closed and convex set,

Received by the editors February 1, 1977.

AMS (MOS) subject classifications (1970). Primary 30A36.

Key words and phrases. Univalent functions, rational functions, domain of univalence, Cakalov-Distler Theorem. 
then the domain of univalence of the family $\mathscr{F}_{3}$ is the complement of $C$.

The director set of a disk $K$ is the Cakalov disk of $K$, and the director set of a line segment $I$ is the disk with $I$ as a diameter. Thus Theorem $C$ contains Theorems $\mathrm{A}$ and $\mathrm{B}$ as special cases. Consequently, we refer to Theorem $\mathrm{C}$ as the Čakalov-Distler Theorem.

In this paper we permit some of the poles to have negative residues. Thus we consider the family of functions $\mathscr{F}=\mathscr{F}\left(K_{1}, K_{2}\right)$, where $f(z)$ is in $\mathscr{F}$ if and only if it can be put in the form

$$
f(z)=\sum_{k=1}^{n} \frac{A_{k}}{z-a_{k}}-\sum_{k=1}^{n} \frac{B_{k}}{z-b_{k}}
$$

where each $a_{k}$ is in $K_{1}$, each $b_{k}$ is in $K_{2}, A_{k} \geqslant 0, B_{k} \geqslant 0$ and $f(z) \neq 0$. Our main result is the determination of maximal domains of univalence of $\mathscr{Y}$ when $K_{1}$ and $K_{2}$ are bounded closed convex sets. It should be observed that in Theorems $\mathrm{A}, \mathrm{B}$, and $\mathrm{C}$ there is only one maximal domain of univalence-the domain of univalence. But in $\mathscr{F}\left(K_{1}, K_{2}\right)$ there may be none, or two distinct maximal domains of univalence.

2. The theorem for two disks. For simplicity, we first assume that $K_{1}$ and $K_{2}$ are closed disks. Since a rotation and translation of the disks will also rotate and translate any domain of univalence, we may assume without loss of generality that the centers $c_{1}$ and $c_{2}$ of $K_{1}$ and $K_{2}$ lie on the real axis and $c_{1}<c_{2}$.

We observe that if $L$ is a straight line through $a$ and $b$ (in $K_{1}$ and $K_{2}$ respectively), then

$$
f(z)=\frac{A}{z-a}-\frac{B}{z-b}, \quad A>0, B>0,
$$

is in $\mathcal{F}$, and we can select $A$ and $B$ so that at any preassigned point $z_{0}$ of $L$ (except $a$ and $b$ ) we can obtain $f^{\prime}\left(z_{0}\right)=0$. Thus all such lines lie outside a domain of univalence of $\mathscr{F}$. If $K_{1} \cap K_{2}$ is not empty then every point in the plane is either on such a line $L$, or is the limit point of points on such lines. It follows that if $K_{1}$ and $K_{2}$ are tangent disks or overlap then there is no domain of univalence for the family $\mathscr{F}$.

TheOrem 1. Let $K_{1}$ and $K_{2}$ be two disjoint closed disks and let $C_{1}$ and $C_{2}$ be the Ċakalov disks of $K_{1}$ and $K_{2}$ respectively. Let $C$ be the union of $C_{1}$ and $C_{2}$ and every line $L$ that meets both $K_{1}$ and $K_{2}$. Then the complement of $C$ consists of two disjoint domains $V^{(+)}$and $V^{(-)}$and each one of these two domains is a maximal domain of univalence of $\mathscr{F}\left(K_{1}, K_{2}\right)$.

Proof. Let $D$ be a maximal domain of univalence. We first consider those points that cannot be in $D$. We have already seen that if $L$ is a line that meets $K_{1}$ and $K_{2}$ then no point of $L$ is in $D$. Further, if $z_{1}$ is a point $C_{1}$ then there is a function 


$$
f(z)=\sum_{k=1}^{n} \frac{A_{k}}{z-a_{k}}
$$

in $\mathscr{F}$ such that $f^{\prime}\left(z_{1}\right)=0$. A similar consideration shows that no point of $C_{2}$ is in $D$. Since the connected set $D$ cannot contain points from both $V^{(+)}$and $V^{(-)}$it follows that either $D \subset V^{(+)}$or $D \subset V^{(-)}$. Without loss of generality we consider the case that $D \subset V^{(+)}$where $V^{(+)}$is contained in the upper half plane.

Let $z_{1}$ and $z_{2}$ be arbitrary distinct points in $V^{(+)}$. To prove that $D=V^{(+)}$ it is sufficient to prove that $Q \neq 0$ where

$$
\begin{aligned}
Q & \equiv \frac{f\left(z_{2}\right)-f\left(z_{1}\right)}{z_{2}-z_{1}} \\
& =-\sum_{k=1}^{n} \frac{A_{k}}{\left(z_{1}-a_{k}\right)\left(z_{2}-a_{k}\right)}+\sum_{k=1}^{n} \frac{B_{k}}{\left(z_{1}-b_{k}\right)\left(z_{2}-b_{k}\right)} .
\end{aligned}
$$

If for some fixed pair $z_{1}, z_{2}$ we have $Q=0$, then

$$
\sum_{k=1}^{n} \frac{A_{k}}{\left(z_{1}-a_{k}\right)\left(z_{2}-a_{k}\right)}=\sum_{k=1}^{n} \frac{B_{k}}{\left(z_{1}-b_{k}\right)\left(z_{2}-b_{k}\right)} .
$$

We will see that equation (6) is impossible because the two sums lie in different sectors of the complex plane (actually one of the sums might be zero, but not the other).

We first observe that in $V^{(+)}$we can define an arg function that is single-valued and continuous. Let

$$
\alpha_{j}=\arg \left(z_{j}-c_{1}\right), \quad \beta_{j}=\arg \left(z_{j}-c_{2}\right)
$$

for $j=1,2$, and for each $a_{k}$ in $K_{1}$ and $b_{k}$ in $K_{2}$ set

$$
\theta_{j k}=\arg \left(z_{j}-a_{k}\right), \quad \varphi_{j k}=\arg \left(z_{j}-b_{k}\right) .
$$

It is easy to see that for $z_{1}, z_{2}$ in $V^{(+)}$we can select these arguments so that $0<\alpha_{j}, \beta_{j}, \theta_{j k}, \varphi_{j k}<\pi$. Next let $\delta_{j}^{(i)}$ be half the angle subtended by $K_{i}$ at $z_{j}(i$, $j=1,2)$. Since $z_{1}$ and $z_{2}$ lie outside $C_{1}$ and $C_{2}$ we have $0 \leqslant \delta_{j}^{(i)}<\pi / 4$, where equality can occur on the left side if some $K$ (and hence also $C$ ) is a single point. With these notations we have the two sets of inequalities

$$
\alpha_{j}-\delta_{j}^{(1)} \leqslant \theta_{j k} \leqslant \alpha_{j}+\delta_{j}^{(1)}
$$

and

$$
\beta_{j}-\delta_{j}^{(2)} \leqslant \varphi_{j k} \leqslant \beta_{j}+\delta_{j}^{(2)},
$$

for $j=1,2$, and $k=1,2, \ldots, n$.

Now consider a typical nonzero term in the sum on the left side of equation (6). By the definitions in (7) and (8) and the inequalities in (9) we have

$$
\begin{aligned}
-\alpha_{1}-\delta_{1}^{(1)}-\alpha_{2}-\delta_{2}^{(1)} & \leqslant \arg \frac{A_{k}}{\left(z_{1}-a_{k}\right)\left(z_{2}-a_{k}\right)} \\
& \leqslant-\alpha_{1}+\delta_{1}^{(1)}-\alpha_{2}+\delta_{2}^{(1)} .
\end{aligned}
$$


Since the extremes in the inequality (11) are independent of $k$, the sum on the left side of (6) lies in the sector defined by (11). Further $2 \delta_{1}^{(1)}+2 \delta_{2}^{(1)}<\pi$, so this sector is contained in a half plane.

The same type considerations applied to a nonzero term on the right side of equation (6) give

$$
\begin{aligned}
-\beta_{1}-\delta_{1}^{(2)}-\beta_{2}-\delta_{2}^{(2)} & \leqslant \arg \frac{B_{k}}{\left(z_{1}-b_{k}\right)\left(z_{2}-b_{k}\right)} \\
& \leqslant-\beta_{1}+\delta_{1}^{(2)}-\beta_{2}+\delta_{2}^{(2)}
\end{aligned}
$$

and hence the sum on the right side is also contained in the sector defined by (12) and this sector is also contained in a half plane.

Now (dropping the subscript $j$ from $z_{j}$ ) a simple geometric argument from the figure shows that for each point $z$ in $V^{(+)}$

$$
\delta^{(1)}+\delta^{(2)}<\beta-\alpha \text { and } \delta^{(1)}+\delta^{(2)}<\pi-(\beta-\alpha) .
$$

Applying these inequalities for $z_{1}$ and $z_{2}$ to the extremes in (11) and (12) we see that

$$
-\beta_{1}+\delta_{1}^{(2)}-\beta_{2}+\delta_{2}^{(2)}<-\alpha_{1}-\delta_{1}^{(1)}-\alpha_{2}-\delta_{2}^{(1)}
$$

(the right side of (12)) $<$ (the left side of (11)), and further

$$
-\alpha_{1}+\delta_{1}^{(1)}-\alpha_{2}+\delta_{2}^{(1)}<-\beta_{1}-\delta_{1}^{(2)}-\beta_{2}-\delta_{2}^{(2)}+2 \pi,
$$

(the right side of $(11))<$ (the left side of $(12))+2 \pi$. Hence the two sectors defined by (11) and (12) are disjoint, except for the common vertex at $z=0$. Since the sums in (6) cannot both be zero, the relation in (6) is impossible. Hence $f(z)$ is univalent in $V^{(+)}$.

We can obtain the same result for $V^{(-)}$by similar considerations, or by applying the part already proved to $\overline{f(\bar{z})}$.

If we allow $K_{1}$ and $K_{2}$ to shrink to distinct points we obtain a trivial but interesting

Corollary. If $a<b, A>0$, and $B>0$ then

$$
f(z)=A /(z-a)-B /(z-b)
$$

is univalent in $\operatorname{Im} z>0$. It is also univalent in $\operatorname{Im} z<0$.

3. An extension. We now replace the disks $K_{1}$ and $K_{2}$ by slightly more general sets and obtain

THEOREM 2. Let $K_{1}$ and $K_{2}$ be two disjoint closed bounded convex sets, and let $C_{1}$ and $C_{2}$ be the director sets of $K_{1}$ and $K_{2}$ respectively. Further let $C$ be the union of $C_{1}, C_{2}$ and every line $L$ that meets both $K_{1}$ and $K_{2}$. Then the complement of $C$ consists of two domains $V^{(+)}$and $V^{(-)}$. If $\mathscr{F}\left(K_{1}, K_{2}\right)$ is the family of all functions of the form (2) with $a_{k}$ in $K_{1}, b_{k}$ in $K_{2}, A_{k} \geqslant 0, B_{k} \geqslant 0$ and $f(z) \not 0$, then each one of the domains $V^{(+)}$and $V^{(-)}$is a maximal domain of univalence for $\mathscr{F}\left(K_{1}, K_{2}\right)$.

Proof. We observe that a disk $K$ has a well-defined center $c$ (see equation 
(7)) but an arbitrary closed convex set does not. However, for each fixed $z$ in $V^{(+)}$we can select a point on the internal bisector of the angle subtended by $K$ at $z$. The selection of $c$ on this bisector serves only to determine the angles $\alpha$ and $\beta$ in (7). With this slight modification the proof given in $\$ 2$ is valid for any two disjoint closed bounded convex sets.

An interesting corollary can be obtained if we let $K_{1}$ and $K_{2}$ be disjoint closed intervals on the real axis, and replace the sums by Stieltjes integrals.

Corollary. If $a<b<c<d$ and $\mu_{1}(t)$ and $\mu_{2}(t)$ are nondecreasing functions then

$$
f(z) \equiv \int_{a}^{b} \frac{1}{z-t} d \mu_{1}(t)-\int_{c}^{d} \frac{1}{z-t} d \mu_{2}(t)
$$

is univalent in $V^{(+)}$, provided the integrals exist and $f(z) \not 0$. Here $V^{(+)}$is the half plane $\operatorname{Im} z>0$ minus the two disks that have the intervals $[a, b]$ and $[c, d]$ as diameters. Further $f(z)$ is univalent in $V^{(-)} \equiv \overline{V^{(+)}}$.

4. Some open questions. We can allow the residues to be complex if they are suitably restricted. Distler [3] extended his Theorem $\mathrm{C}$ to a family of functions of the form (1) where each $A_{k}$ lies in the sector $\left|\arg A_{k}\right| \leqslant \sigma<\pi / 4$ and with a suitably modified definition of the director set, Distler found the domain of univalence of this larger family.

It is possible to extend Theorems 1 and 2 in the same manner, but if complex residues are permitted, then the lines $L$ that meet $K_{1}$ and $K_{2}$ must be replaced by certain circles (where $f^{\prime}(z)$ may be zero if $f(z)$ is the example function (3)). Hence the maximal domains of univalence are greatly reduced, and the resulting theorem is more involved (and perhaps less interesting).

A more difficult problem, and more interesting would be to find a maximal domain of $p$-valence of the family $\mathscr{F}$ for each integer $p>1$, for any of the families considered so far.

Further one might consider $m$ mutually disjoint bounded closed convex sets $K_{1}, K_{2}, \ldots, K_{m}$ together with $2 m$ constants $\theta_{1}, \theta_{2}, \ldots, \theta_{m}, \sigma_{1}$, $\sigma_{2}, \ldots, \sigma_{m}$. Here $\mathscr{F}$ is the family of functions

$$
f(z)=\sum_{j=1}^{m} \sum_{k=1}^{n} \frac{A_{j k}}{z-a_{j k}}
$$

where each $a_{j k}$ is in $K_{j}$ and the nonzero residues satisfy the inequalities

$$
\theta_{j}-\sigma_{j} \leqslant \arg A_{j k} \leqslant \theta_{j}+\sigma_{j}
$$

for $j=1,2, \ldots, m$ and $k=1,2, \ldots, n$. Under what conditions on the sets $K_{j}$ and the constants $\theta_{j}, \sigma_{j}$ does the family have a maximal domain of univalence (or $p$-valence), and if one exists what is it?

One might also ask for a domain of univalence if the poles of order 1 are replaced by $m$ th order poles $(m>1)$ in any one of the families considered so far. It is easy to extend Theorem 2 in this direction to find a domain of univalence for $F$, but it seems to be difficult to find a maximal domain of univalence in this case. 
Finally we observe that if $K$ is a discrete set of points (rather than a convex set) then the family described in Theorem $\mathrm{C}$ may have more than one maximal domain of univalence. Some interesting results in the case that $K$ is a set with more than one component have been obtained by S. H. Lameier [4].

\section{REFERENCES}

1. L. Ċakalov, Sur une classe de fonctions analytiques univalentes, C. R. Acad. Sci. Paris 242 (1956), 437-439.

2. Sur les domaines d'univalence de certaines classes de fonctions analytiques, B"lgar. Akad. Nauk Izv. Mat. Inst. 4 (1960), No. 2, 43-55. (Bulgarian, Russian and French summaries).

3. R. J. Distler, The domain of univalence of certain classes of meromorphic functions, Proc. Amer. Math. Soc. 15 (1964), 923-928.

4. S. Lameier, On domains of univalence of certain meromorphic functions, Master's Thesis, University of Cincinnati, Cincinnati, Ohio.

Department of Mathematics, University of South Florida, Tampa, Florida 33620 